\title{
Prediction of GOLD Stage in Patients Hospitalized with COPD Exacerbations with Blood Neutrophils and Demographic Parameters as Risk Factors
}

Jing Chen ( $D$ 695347385@qq.com )

Chinese Academy of Science

Guo Liquan

Chinese Academy of Sciences

Xiong Daxi

Chinese Academy of Sciences

Yuan Qun

Nanjing Medical University

Yang Zhao

Nanjing Medical University

Research

Keywords: GOLD stage, ECOPD, Blood NEU\%, Demographic parameters

Posted Date: January 21st, 2021

DOI: https://doi.org/10.21203/rs.3.rs-150711/v1

License: (c) (i) This work is licensed under a Creative Commons Attribution 4.0 International License. Read Full License 


\section{Abstract \\ Background}

Patients hospitalized with chronic obstructive pulmonary disease (COPD) exacerbations are unable to complete the pulmonary function test reliably due to poor health status. Creating an easy-to-use instrument to identify the Global Initiative for Chronic Obstructive Lung Disease (GOLD) stage could offer valuable information that assist clinicians to choose appropriate clinical care to decrease the mortality in these patients. The objective of this study was to develop a prediction model to identify the GOLD stage in the hospitalized exacerbation of chronic obstructive pulmonary disease (ECOPD) patients.

\section{Methods}

This study involved 155 patients hospitalized for ECOPD. All participants completed lung function tests and the collection of blood neutrophils and demographic parameters. The area under the receiver operating characteristic (ROC) curve was used to confirm the disease severity predictive ability of blood neutrophils and demographic parameters. A support vector regression (SVR) based GOLD stage prediction model was created in the training set (75\%) and applied to the testing set $(25 \%)$.

\section{Results}

The percentage of neutrophils (NEU\%) combined with the demographic parameters was associated with a higher risk to severe episode of ECOPD. The area under the ROC curve was 0.84 . The prediction model could predict the GOLD stage with acceptable prediction accuracy. The root-mean-square error (RMSE) of the forced expiratory volume in one second as the percentage of the predicted (FEV ${ }_{1} \%$ pred) was $8.84 \%$. The accuracy of the GOLD stage prediction was $90.24 \%$.

\section{Conclusions}

The NEU\% and demographic parameters are associated with the pulmonary function of the hospitalized ECOPD patients. The established prediction model could assist clinicians to diagnose GOLD stage and establish appropriate clinical care.

\section{Background}

Chronic obstructive pulmonary disease (COPD) is a progressive lung condition and a leading cause of adult morbidity and mortality worldwide 1,2,3. Exacerbation of chronic obstructive pulmonary disease (ECOPD) is an event characterized by a sustained worsening of the respiratory symptoms of a patient (including cough, phlegm production, and dyspnea), beyond normal day-to-day variations, which results in additional therapy 4, 5. These episodes requiring hospitalization are associated with increased 
morbidity, mortality and enormous burden upon healthcare systems 6,7 . Inflammation is a key component in the pathogenesis of COPD 8. It has previously been observed that COPD is not only associated with abnormal inflammatory response of the lung, but also with systemic inflammation, including systemic oxidative stress, activation of circulated immune cells and inflammatory cells, and the increased inflammatory cytokines circulating levels 9 . It is generally considered that ECOPD reflects a flare-up of these underlying inflammatory processes 10 , and is linked to a neutrophilic signature response 11.

A recent systematic literature review concluded that ECOPDs are extremely dangerous events.There is an urgent need to identify tolerable treatment guidelines and manage acute exacerbations in hospitalized ECOPD patients 12. The Global Initiative for Chronic Obstructive Lung Disease (GOLD) guidelines classify COPD based on the value of forced expiratory volume in one second as percentage of predicted ( $F E V_{1} \%$ pred) as shown in Table 1. GOLD stage $\mathbb{X}-\mathbb{Q}$ represents mild, moderate, severe and very severe. Inadequate diagnosis of COPD and the lack of spirometric assessment can lead to inadequate treatment strategies, with health costs and risks for patients, leading to delays in diagnosing and treatment of the true cause of the symptoms $13,14,15$. Patients hospitalized with COPD exacerbations due to poor health status, are unable to complete the pulmonary function test reliably, as one longitudinal study indicated, $50 \%$ of pulmonary function test results are unacceptable 16 .

Table 1

GOLD stage of COPD

\begin{tabular}{|ll|}
\hline FEV $_{1} \%$ predicted (\%) \\
\hline GOLD $\otimes$ & $\geq 80$ \\
\hline GOLD $\otimes$ & $50-79$ \\
\hline GOLD $\otimes$ & $30-49$ \\
\hline GOLD $\otimes$ & $<30$ \\
\hline
\end{tabular}

Many studies evaluating disease severity in COPD patients have focused on the stable stage instead of exacerbating stage. Based on the needs of unified assessment criteria that can accurately reflect the pulmonary function of hospitalized ECOPD patients, we explored the predictive ability of blood percentage of neutrophils (NEU\%) and demographic parameters in GOLD stage and created a prediction model based on support vector regression (SVR) for predicting GOLD stage in Hospitalized ECOPD Patients 17.

\section{Materials And Methods}

\section{Subjects selection}

A total of 155 subjects ( 135 males and 20 females) were included in the study, all of whom were from the Respiratory Department of The Affiliated Suzhou Science and Technology Town Hospital of Nanjing 
Medical University in Suzhou, China. With the permission of the Local Ethics Committee, the study was conducted between January 2018 and March 2020. 241 patients were collected from initially selected patients hospitalized for ECOPD, who were over 40 years old, clinically diagnosed as COPD, patients with aggravating symptoms and with no history of pulmonary dysfunction. A total of 86 patients were excluded due to the following exclusion criteria: (1) 34 patients with noninfectious exacerbations; (2) 30 patients withdrew consent; (3) 22 patients who have mechanical barrier or hearing disease. Ultimately, 155 patients were enrolled (Fig. 1).

\section{Clinical data}

Demographic information including gender, age, height and weight was recorded upon admission to the hospital. After interview and written consent from participants, 155 patients participated in pulmonary function tests using equipment manufactured by CareFusion, USA. Tests are guided by the same professional doctor when the patients' health status permitted. Three effective pulmonary function tests were performed with the same, regularly gauged spirometer to reduce measurement errors. The average $\mathrm{FEV}_{1}$ \%pred value and the GOLD stage of the three tests was recorded. FEV 1 was reported in litres and Zscore to correctly characterize the cohorts investigated. The FEV $\mathrm{Z}_{1}$-score of each patients was derived by using norms from the Global Lung Initiative (GLI) basing specially on developed software 18. Peripheral venous blood was drawn for peripheral blood examination at the same time period with pulmonary function tests. The automatic blood analyzer (SYSMEX Japan) was used to calculate the NEU\%.

\section{Risk stratification}

As $\mathrm{FEV}_{1}<1 \mathrm{~L}$ indicates that the impact of disease on patients' daily life and health is increasing rapidly, most physicians, technologists and radiologists adopt $1 \mathrm{~L}$ as a threshold for $\mathrm{FEV}_{1}$ to evaluate pulmonary function 19,20. All enrolled patients were assessed whether they have severe episodes of ECOPD using $\mathrm{FEV}_{1}<1 \mathrm{~L}$ as the threshold of severe episode of ECOPD and were classified into four stages according to GOLD guidelines.

\section{Statistical analysis}

Demographic information, the blood NEU\% and $\mathrm{FEV}_{1}$ of all the participants were expressed as the mean (SD) or median (IQR) for normally or non-normally distributed data respectively and the percentages for the count data. Differences of continuous variables between Gold stages were assessed using Student's t-tests or Mann-Whitney U tests. ANOVA or Kruskal-Wallis test was used to compare more than two groups. Developing univariate logistic regression models to assess the correlation between NEU\%, demographic parameters and severe episode of ECOPD. All variables correlated with severe episode of ECOPD were considered in a multivariable model. Receiver operating characteristic (ROC) curves were constructed to evaluate the discrimination of models. An area under the ROC curve of 0.8 or greater is generally considered to be a good predictor 21 . 
The outcome variables were defined as the $\mathrm{FEV}_{1} \%$ pred value and the GOLD stage of patients hospitalized for ECOPD. The patients were randomly divided into a training set $(75 \%)$ and a testing set $(25 \%)$. The training set was used to develop the SVR based prediction model, whereas the testing set was used to validate the predictive performance of $\mathrm{FEV}_{1} \%$ pred value and GOLD stage. Pearson correlation coefficient was adopted to evaluate the linear correlation of the predicted and the measured values of $\mathrm{FEV}_{1} \%$ pred. If the P-value is less than 0.05 , the difference is considered significant. The root mean squared errors (RMSE) and correlation coefficient ( $r$ ) were used to quantitatively describe the strength of the relationship between the predicted and the measured values of $\mathrm{FEV}_{1} \%$ pred. The GOLD stage was classified by the predicted values of $\mathrm{FEV}_{1}$ \% pred according to the GOLD guidelines, and compared with the Gold stage classified by the measured value of $\mathrm{FEV}_{1} \%$ pred. We will also report the GOLD stage prediction accuracy to assess the discrimination ability of the model.

\section{Results}

\section{Demographic information, blood NEU\% and $\mathrm{FEV}_{1}$ in all the enrolled patients}

Ultimately, 155 subjects were enrolled. A total of 93 subjects were defined as non-severe episode pattern, and 62 subjects were defined as severe episode pattern. The height, weight, BMI, FEV ${ }_{1}$, and $\mathrm{FEV}_{1} \%$ Pred in the non-severe episode group were higher than those in the severe episode group, with significance $(\mathrm{p}<$ 0.05). Blood NEU\% in the non-severe episode group was lower than that in the severe episode group, with significance $(p<0.001)$. There was no significant difference in the gender and age between the two groups $(P=0.587, P=0.202)$ (Table 2$)$.

Table 2 Comparison of parameters between non-severe episode group and severe episode group in all enrolled subjects 


\begin{tabular}{|c|c|c|c|c|c|}
\hline \multirow[t]{2}{*}{ Category } & \multirow[t]{2}{*}{ Variable } & \multirow[t]{2}{*}{ Units } & \multicolumn{3}{|c|}{$\mathrm{FEV}_{1}<1 \mathrm{~L}$ as the threshold of severe episode of ECOPD } \\
\hline & & & $\begin{array}{l}\text { Non-severe episode } \\
(\mathrm{N}=93)\end{array}$ & $\begin{array}{l}\text { Severe episode } \\
(\mathrm{N}=62)\end{array}$ & $\begin{array}{l}\mathrm{P} \\
\text { value }\end{array}$ \\
\hline \multirow[t]{5}{*}{ Demographics } & Male & $N(\%)$ & $82(88.17)$ & $53(85.48)$ & 0.587 \\
\hline & Age & Years & $74.00(68.00,79.00)$ & $76.50(69.00,80.00)$ & 0.202 \\
\hline & Height & $\mathrm{cm}$ & $\begin{array}{l}165.50(162.00 \\
170.00)\end{array}$ & $\begin{array}{l}\text { 163.50(157.50, } \\
168.00)\end{array}$ & 0.018 \\
\hline & Weight & $\mathrm{kg}$ & $66.57(9.94)$ & $59.79(10.31)$ & $<0.001$ \\
\hline & $\mathrm{BMI}$ & & $24.38(3.73)$ & $22.65(3.36)$ & 0.008 \\
\hline Blood count & NEU & $\%$ & $72.05(65.08,80.95)$ & $82.40(71.80,86.40)$ & $<0.001$ \\
\hline \multirow{3}{*}{$\begin{array}{l}\text { Pulmonary } \\
\text { function }\end{array}$} & $\mathrm{FEV}_{1}$ & L & $1.37(1.15,1.72)$ & $0.78(0.67,0.86)$ & $<0.001$ \\
\hline & $\begin{array}{l}\mathrm{FEV}_{1} \mathrm{Z}- \\
\text { score }\end{array}$ & & $-2.29(-2.70,-1.83)$ & $-3.53(-3.77,-3.18)$ & $<0.001$ \\
\hline & $\mathrm{FEV}_{1} \%$ Pred & & $57.34(49.50,67.95)$ & $33.46(28.83,37.46)$ & $<0.001$ \\
\hline
\end{tabular}

Data expressed as mean (SD) or median (IQR); N: numbers of patients

All enrolled patients were classified into GOLD stage $\mathbb{X}$ based on the GOLD guidelines. The factors associated with GOLD stage are shown in Table 3. Univariable analysis demonstrated that gender, age, weight, BMI and NEU \% are the risk factors of different GOLD stage (Table 3). On the basis of the univariate analysis, the univariable and multivariable models are used to discriminate a severe episode of ECOPD.

Table 3 Characteristic distribution comparison among different COPD GOLD stage groups 


\begin{tabular}{|c|c|c|c|c|c|}
\hline \multirow[t]{2}{*}{ Characteristic } & \multicolumn{4}{|c|}{ COPD GOLD stage } & \multirow[t]{2}{*}{$P$ value } \\
\hline & $\varangle(N=13)$ & $\nabla(N=60)$ & $\otimes(N=66)$ & $\rrbracket(N=16)$ & \\
\hline Gender & & & & & 0.032 \\
\hline Male & $10(7.41 \%)$ & $50(37.04 \%)$ & $60(44.44 \%)$ & $15(11.11 \%)$ & \\
\hline Female & $3(15 \%)$ & $11(55 \%)$ & $5(25 \%)$ & $1(5 \%)$ & \\
\hline Age & & & & & 0.001 \\
\hline$<60$ & $2(15.38 \%)$ & $10(76.92 \%)$ & $1(7.69 \%)$ & $0(0 \%)$ & \\
\hline $60-69$ & $4(7.84 \%)$ & $24(47.06 \%)$ & $18(35.29 \%)$ & $5(9.80 \%)$ & \\
\hline $70-79$ & $5(8.62 \%)$ & $21(36.21 \%)$ & $28(48.28 \%)$ & $4(6.90 \%)$ & \\
\hline$\geq 80$ & $2(6.06 \%)$ & $6(18.18 \%)$ & $18(54.55 \%)$ & $7(21.21 \%)$ & \\
\hline Height & & & & & 0.101 \\
\hline$<155$ & $2(16.67 \%)$ & $7(58.33 \%)$ & $3(25 \%)$ & $0(0 \%)$ & \\
\hline $155-164$ & $4(6.67 \%)$ & $21(35 \%)$ & $27(45 \%)$ & $8(13.33 \%)$ & \\
\hline $165-174$ & $7(9.46 \%)$ & $30(40.54 \%)$ & $30(40.54 \%)$ & $7(9.46 \%)$ & \\
\hline$\geq 175$ & $0(0 \%)$ & $3(33.33 \%)$ & $5(55.56 \%)$ & $1(11.11 \%)$ & \\
\hline Weight & & & & & 0.035 \\
\hline$<50$ & $0(0 \%)$ & $5(26.32 \%)$ & $13(68.42 \%)$ & $1(5.26 \%)$ & \\
\hline $50-59$ & $1(3.13 \%)$ & $11(34.38 \%)$ & $19(59.38 \%)$ & $1(3.13 \%)$ & \\
\hline $60-69$ & $3(4.84 \%)$ & $28(45.16 \%)$ & $20(32.26 \%)$ & $11(17.74 \%)$ & \\
\hline$\geq 70$ & $9(21.43 \%)$ & $17(40.48 \%)$ & $13(30.95 \%)$ & $3(7.14 \%)$ & \\
\hline BMI & & & & & 0.038 \\
\hline$<18.5$ & $0(0 \%)$ & $6(30 \%)$ & $13(65 \%)$ & $1(5 \%)$ & \\
\hline $18.5-24.5$ & $3(4 \%)$ & $28(37.33 \%)$ & $35(46.67 \%)$ & $9(12 \%)$ & \\
\hline $24.5-30$ & $9(17.31 \%)$ & $23(44.23 \%)$ & $14(26.92 \%)$ & $6(11.54 \%)$ & \\
\hline$>30$ & $1(12.50 \%)$ & $4(50 \%)$ & $3(37.50 \%)$ & $0(0 \%)$ & \\
\hline NEU\% & & & & & $<0.001$ \\
\hline$<70$ & $9(15.25 \%)$ & $30(50.85 \%)$ & $19(32.20 \%)$ & $1(1.69 \%)$ & \\
\hline $70-80$ & $3(6.98 \%)$ & $18(41.86 \%)$ & $17(39.53 \%)$ & $5(11.63 \%)$ & \\
\hline$>80$ & $1(1.89 \%)$ & $13(24.53 \%)$ & $29(54.72 \%)$ & $10(18.87 \%)$ & \\
\hline
\end{tabular}


$\mathrm{N}$ : numbers of patients

\section{Discrimination of a severe episode of ECOPD}

ROC plots and the areas under the ROC curves of the various models to discriminate a severe episode of ECOPD are shown in Fig.2 and Table 4. A model incorporating NEU\% had an area under the ROC curve of 0.68. The final model including all proved risk factors (Model 7) identified in the multivariable analysis showed good discrimination with an area under the ROC curve of 0.84 . The area under the ROC curve of the final model without NEU\% (Model 6 ) was 0.77 , indicating that NEU\% adds discrimination accuracy as a biomarker.

Table 4 The areas under the ROC curves of univariable and multivariable models

\begin{tabular}{|lll|}
\hline Model & The areas under the ROC $(95 \% \mathrm{Cl})$ & P value \\
\hline Model 1 & $0.52(0.41$ to 0.62$)$ & 0.760 \\
\hline Model 2 & $0.57(0.47$ to 0.67$)$ & 0.202 \\
\hline Model 3 & $0.65(0.56$ to 0.75$)$ & $<0.001$ \\
\hline Model 4 & $0.63(0.53$ to 0.73$)$ & 0.013 \\
\hline Model 5 & $0.68(0.59$ to 0.78$)$ & $<0.001$ \\
\hline Model 6 & $0.77(0.68$ to 0.85$)$ & $<0.001$ \\
\hline Model 7 & $0.84(0.77$ to 0.89$)$ & $<0.001$ \\
\hline
\end{tabular}

\section{GOLD stage prediction}

The characteristics of the training set and testing set are included in Table 5. There was no significant difference in all the involved factors, i.e., demographics, blood count, pulmonary function and COPD GOLD stage of subjects between the two groups. Fig. 3 and Fig. 4 show the predictive ability in $\mathrm{FEV}_{1} \%$ pred value of the SVR based prediction model. The association between the predicted and the measured $\mathrm{FEV}_{1} \%$ pred value was strong with $r=0.92$; and the difference is not significant $(P>0.05)$.

The total sample size of GOLD stage I is 13, most hospitalized ECOPD patients tend to have higher GOLD stage. The degree of airflow limitation of patients with GOLD stage I and II is moderate, we combined GOLD stage I and GOLD stage II as the moderate group. The predictive performance on the $\mathrm{FEV}_{1} \%$ pred value and GOLD stage was shown in Table 6. Fig.4 indicated that in the case of FEV $\mathrm{Fpred}_{1}$ exceeding $70 \%$, the model may bring pessimistic prediction results. Analysis on the GOLD stage predictive performance showed that, under the circumstance of predicted $\mathrm{FEV}_{1} \%$ pred exceeding $70 \%$, the algorithm would overestimate the GOLD stage. To be more specific, patients of GOLD stage \may be classified to 
GOLD stage $\rrbracket$. GOLD guidelines defines GOLD stage I and GOLD stage \as moderate airflow obstruction, and the treatment plan will not be confused with GOLD stage $\nabla$ and GOLD stage $\otimes$, which stand for severe airflow obstruction. Fig. 5 shows the predictive performance in different GOLD stage. The overall COPD GOLD stage prediction accuracy was $90.24 \%$.

Table 5 Characteristic of the training set and the testing set

\begin{tabular}{|c|c|c|c|c|c|}
\hline Category & Variable & Units & $\begin{array}{l}\text { Training set } \\
(\mathrm{N}=114)\end{array}$ & Testing set $(\mathrm{N}=41)$ & $P$ value \\
\hline \multirow[t]{4}{*}{ Demographics } & Male & $\mathrm{N}(\%)$ & $101(88.60)$ & $34(82.93)$ & 0.428 \\
\hline & Age & years & $76.00(68.00,80.00)$ & $\begin{array}{l}71.00(69.00 \\
78.00)\end{array}$ & 0.673 \\
\hline & Weight & $\mathrm{kg}$ & 63.46(10.15) & 63.23(12.12) & 0.917 \\
\hline & BMI & $\mathrm{kg} / \mathrm{m} 2$ & $23.54(3.68)$ & $23.66(3.63)$ & 0.869 \\
\hline Blood count & NEU & $\%$ & $76.50(66.60,84.25)$ & $\begin{array}{l}78.40(69.90 \\
84.30)\end{array}$ & 0.385 \\
\hline $\begin{array}{l}\text { Pulmonary } \\
\text { function }\end{array}$ & $\mathrm{FEV}_{1} \%$ Pred & $\%$ & $46.59(34.57,59.34)$ & $\begin{array}{l}47.66(32.64 \\
59.30)\end{array}$ & 0.822 \\
\hline \multirow[t]{4}{*}{ COPD GOLD Stage } & $\square$ & $\mathrm{N}(\%)$ & $9(7.89)$ & $4(9.76)$ & 0.603 \\
\hline & प & $\mathrm{N}(\%)$ & $49(42.98)$ & 13(31.72) & \\
\hline & प & $\mathrm{N}(\%)$ & 43(37.72) & $21(51.22)$ & \\
\hline & प & N (\%) & $13(11.40)$ & $3(7.32)$ & \\
\hline
\end{tabular}

$\mathrm{N}$ : numbers of patients

Table 6 Results of the prediction model in the testing sets

\begin{tabular}{|c|c|c|c|}
\hline \multirow{2}{*}{\multicolumn{2}{|c|}{ Model evaluation }} & RMSE of $\mathrm{FEV}_{1} \%$ Pred & Accuracy of COPD GOLD stage \\
\hline & & & $(\%)$ \\
\hline \multicolumn{2}{|c|}{$\begin{array}{l}\text { Total population of test set } \\
(\mathrm{N}=41)\end{array}$} & 8.84 & 90.24 \\
\hline \multirow{3}{*}{$\begin{array}{l}\text { COPD GOLD } \\
\text { Stage }\end{array}$} & $\operatorname{Vand} \nabla(\mathrm{N}=17)$ & 12.51 & 94.11 \\
\hline & $\nabla(N=21)$ & 4.98 & 90.47 \\
\hline & $\nabla(\mathrm{N}=3)$ & 2.83 & 66.67 \\
\hline
\end{tabular}

$\mathrm{N}$ : numbers of patients 


\section{Discussion}

ECOPD is a kind of acute attack process, patients' respiratory symptoms continue to worsen over their

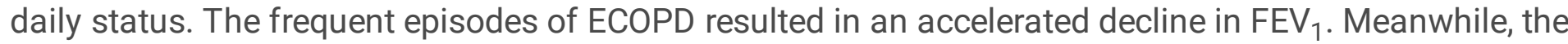
rapid decline of $\mathrm{FEV}_{1}$ performs as an independent hazard factor for ECOPD. The vicious circle between the decline of $\mathrm{FEV}_{1}$ and the frequent attack of ECOPD affect the prognosis and mortality of patients 22 . In this analysis, we focused on the discrimination value of blood NEU\% as a biomarker for a severe episode of ECOPD, and the GOLD stage prediction in hospitalized ECOPD patients. We hoped to create an easy-to-use measure to estimate the value of $\mathrm{FEV}_{1} \%$ Pred and identify the GOLD stage that could assist clinicians to choose appropriate measures of medical care to decrease future hospitalization rates and mortality in hospitalized ECOPD patients.

In line with previous studies, the end of pulmonary function test relied on the cooperation of ECOPD patients, most likely due to the limitation by force-velocity characteristics of expiratory muscles 16, 23 . Biomarkers are required for effective risk stratification and making individualized treatment decision.

The pathophysiological mechanisms of most cases of ECOPD is an acute burst of local or systemic inflammatory mediators and respiratory bacterial or virus infection. Usually, high levels of non-specific inflammatory biomarkers are expected 24 . Neutrophils are the most abundant inflammatory cells in blood and sputum, and as neutrophil proteases can generalize many of the characteristics of ECOPD including emphysema and mucus hypersecretion 25, ECOPD is characterized as a neutrophil inflammatory disorder in most cases. A study on peripheral blood neutrophils from ECOPD patients conducted by Milara et al showed that compared with healthy control group, the release of the neutrophil activation marker neutrophil elastase (NE) and reactive oxygen species (ROS)increased by 2 times and $30 \%$ respectively 26 . Jones et al observed that compared with the healthy controls, bacterial stimulated neutrophil degranulation was greater in the ECOPD group 27. Corhay et al focused on exacerbation whichever its trigger and found that that neutrophil inflammatory markers declined after treatment 28 . We designated a statistically significant difference in the NEU\% between ECOPD patients with different GOLD stages to extend these findings. ECOPD patients with higher blood NEU\% had a higher tendency of severe episode of ECOPD, and the GOLD stage risk stratification may be higher. This differences between ECOPD patients with different GOLD stage is consistent with the results of Perera et al. They found that there were significant differences in systemic markers of inflammation between patients with GOLD stages III and IV vs. controls without COPD, while there was no significant difference between GOLD II patients and controls 29.

We sought for factors that would discriminate a severe episode of ECOPD in clinical cases. Although the multivariable demographics parameters or NEU\% reflected the relative risk of a severe episode of ECOPD, considering the moderate values of areas under the ROC curves, the overall prediction ability is still quite limited. No matter which cut-off level is chosen, the false positive rate is still very high, so the specificity for acceptable value of sensitivity is low. With increase in blood NEU\%, the risk of a severe episode of 
ECOPD increased. The overall discrimination value of multivariable factors including demographics parameters and blood NEU\% was encouraging in view of the 0.84 value of area under the ROC curve.

To further study the FEV ${ }_{1} \%$ Pred predictive and the GOLD stage categorization ability of the blood NEU\% and demographic parameters, we randomly divided ECOPD patients into a training set and a testing set to develop a prediction model, whereas the testing set was used to validate the predictive performance of $\mathrm{FEV}_{1} \%$ pred value and GOLD stage. The demographic parameters selected including several with demonstrated capacity: gender, age, weight, BMI. We used supervised learning algorithm to evaluate the predictive ability of the risk factors and classified the subjects to 4 different GOLD stages. Searching for the right subjects is one of the major difficulties of our study. Support vector machine (SVM) is a learning method based on the principle of structural risk minimization of statistical learning theory. It shows many unique advantages in solving the problem of small sample and nonlinear 30. SVR is a model dealing with the SVM regression problems, which shows acceptable regression and classification capacity in estimating the value of $\mathrm{FEV}_{1} \%$ Pred and identifying the GOLD stage.

To our knowledge, this is the first study in ECOPD patients to predict the value of FEV ${ }_{1} \%$ Pred and identify the GOLD stage based on demographic parameters and blood NEU\%. In the absence of a clear biomarker to categorize the GOLD stage of ECOPD patients, our research provides an auxiliary guidance value for the clinicians to diagnose GOLD stage and establish appropriate clinical care as the demographics parameters and blood NEU\% are easy to be obtained.

Limitations of our study also should be noted. First, the relatively small number of subjects enrolled in this study may limit the predictive performance of the model, especially when comparing to the previous work of Cristóbal et al. 31 and Godtfredsen and coworkers 32. The predictive ability of prediction model is limited in ECOPD patients with optimistic degree of airflow obstruction, this may also result from the lower influence of inflammatory factors when the symptoms are moderate. To find proper ECOPD patients and guide them to complete the pulmonary function test is one of the biggest difficulties during our research. To overcome this limitation, we used the most widely accepted learning method SVM to establish prediction model. The grouping strategy of training set and testing set can tackle the problem of multiple covariates larger than the samples (patients) or ' $p>n$ problem'. Importantly, the overall ECOPD GOLD stage prediction accuracy of the establish prediction model was $90.24 \%$. Secondly, Sørheim and coworkers showed that pulmonary function injury may differ from gender. There is a gender imbalance in our study cohort as the ECOPD patients included were mostly male (135/155). The model's predictive performance on female patients may be limited. Therefore, our future work is to balance the sex ration and extend the observation time to carry out larger scale research to verify our findings. As an additional limitation of the study, patient's general condition, comprehension and cooperative degree could influence accuracy of pulmonary function test result. Nevertheless, every enrolled patients were trained and guided by the same professional physician to minimize the impact of external factors on the measurement.

\section{Conclusions}


In summary, a prediction model based on demographic parameters and blood NEU\% is established to predict the value of $\mathrm{FEV}_{1} \%$ Pred and identify the GOLD stage for patients hospitalized with ECOPD. This easy-to-use instrument could assist clinicians to diagnose GOLD stage and offer valuable information about the appropriate clinical care for hospitalized ECOPD patients.

\section{Abbreviations}

COPD

Chronic Obstructive Pulmonary Disease

GOLD

The Global Initiative for Chronic Obstructive Lung Disease

ECOPD

Exacerbation of Chronic Obstructive Pulmonary Disease

ROC

The Receiver Operating Characteristic

SVR

Support Vector Regression

NEU\%

The Percentage of Neutrophils

\section{RMSE}

The Root-mean-square Error

FEV $1 \%$ pred

The Forced Expiratory Volume in One Second as the Percentage of the Predicted

GLI

The Global Lung Initiative

ROS

Reactive Oxygen Species

NE

Neutrophil Elastase

SVM

Support Vector Machine

\section{Declarations}

\section{Acknowledgments}

We are grateful for the physicians, clinicians, and staff members of the various services of The Affiliated Suzhou Science and Technology Town Hospital of Nanjing Medical University We also gratefully acknowledge the patients who participate in the study.

\section{Funding}


This study was funded by the grants from the Suzhou Special Technical Project for Diagnosis and Treatment of Key Clinical Diseases (No. LCZX201931).

Author information

Affiliations

School of Biomedical Engineering (Suzhou), Division of Life Sciences and Medicine, University of Science and Technology of China, Hefei 230026, P. R. China

Jing Chen, Li-quan Guo, Da-xi Xiong

Suzhou Institute of Biomedical Engineering and Technology, Chinese Academy of Sciences, Suzhou 215163, P. R. China

Jing Chen, Li-quan Guo, Da-xi Xiong

Respiratory Department, The Affiliated Suzhou Science and Technology Town Hospital of Nanjing Medical University, Suzhou 215163, P. R. China

Zhao Yang, Qun Yuan

Corresponding author:

Correspondence to Da-xi Xiong and Li-quan Guo.

\section{Author contributions statement}

J.C. conceived and designed the study, acquired and analyzed the data, and wrote the manuscript. Z.Y. and Q.Y. contributed to data analysis. D.X.X. provided support for statistical analysis, interpretation, and critical revision of the manuscript for important intellectual content. L.Q.G. helped to conceptualize and design the study. All authors have read and approved the manuscript.

Ethics approval and consent to participate

The study was approved by the local ethics committee approval number 18/ECA/0012. All patients agree to participate and signed a consent form.

Consent for publication

Not applicable.

Availability of data and materials

Please contact authors for data requests. 


\section{Competing interests}

The authors declare no competing interests.

\section{Rights and permissions}

Open Access This article is distributed under the terms of the Creative Commons Attribution 4.0 International License (http://creativecommons.org/licenses/by/4.0/), which permits unrestricted use, distribution, and reproduction in any medium, provided you give appropriate credit to the original author(s) and the source, provide a link to the Creative Commons license, and indicate if changes were made. The Creative Commons Public Domain Dedication waiver (http://creativecommons.org/publicdomain/zero/1.0/) applies to the data made available in this article, unless otherwise stated.

\section{References}

1 GOLD. Global strategy for the diagnosis, management and prevention of COPD, global initiative for chronic obstructive lung disease (GOLD);2019. [Update 2019] Available from: http://goldcopd.org. Accessed February 5, 2019

2 Adeloye D, Chua S, Lee C, Basquill C, Papana A, Theodoratou E, et al. Global and regional estimates of COPD prevalence: Systematic review and meta-analysis. J Glob Health 2015; 5:020415.doi: 10.7189/jogh.05-020415

3 Projections of mortality and causes of death, 2015 and 2030. Geneva: World Health Organization; Available from: http://www.who.int/healthinfo/global_burden_disease/projections/en/ (Accessed on September 11, 2017).

4 Burge S, Wedzicha JA. COPD exacerbations: definitions and classifications. Eur Respir J Suppl. 2003;41:46s-53s.

5 Rubinsztajn R, Przybylowski T, Maskey-Warzechowska M, Karwat K, Chazan R. Exacerbations of chronic obstructive pulmonary disease and quality of life of patients. Adv Exp Med Biol. 2016;884:69-74.

6 Donaldson GC, Seemungal TAR, Bhowmik A, Wedzicha JA. Relationship between exacerbation frequency and lung function decline in chronic obstructive pulmonary disease. Thorax. 2002;57:847-852.

7 Schmidt SAJ, Johansen MB, Olsen M, Xu X, Parker JM, Molfino NA, Lash TL, Sørensen HT, Christiansen $\mathrm{CF}$. The impact of exacerbation frequency on mortality following acute exacerbations of COPD: a registrybased cohort study. BMJ Open. 2014;4:e006720.

8 Rhee C K, Kim D K. Role of phosphodiesterase-4 inhibitors in chronic obstructive pulmonary disease[J]. Korean J Intern Med, 2020, 35(2):276-283. 
9 Agusti A G N, Noguera A, Sauleda J , et al. Systemic effects of chronic obstructive pulmonary disease[J]. Eur Respir J, 2003, 21(2):347-360.

10 Jing Z , Chun C, Ning S, et al. Systemic Inflammatory Marker CRP Was Better Predictor of Readmission for AECOPD Than Sputum Inflammatory Markers[J]. Arch Bronconeumol (English Edition), 2016.

$11 \mathrm{Gao} J$, Chen B , Wu S, et al. Blood cell for the differentiation of airway inflammatory phenotypes in COPD exacerbations[J]. BMC Pulm Med, 2020, 20.

12 Angela, M, Abbatecola, et al. Practical management problems of chronic obstructive pulmonary disease in the elderly: acute exacerbations.[J]. Curr Opin Pulm Med, 2011.

13 Fernandez-Villar A, Soriano JB, Lopez-Campos JL. Overdiagnosis of COPD: precise definitions and proposals for improvement. Br J Gen Pract. 2017;67:183-4.

14 Hartl S, Lopez-Campos JL, Pozo-Rodriguez F, Castro-Acosta A, Studnicka M, Kaiser B, et al. Risk of death and readmission of hospital-admitted COPD exacerbations: European COPD Audit. Eur Respir J. 2016;47:113-21.

15 Wu H, Wise RA, Medinger AE. Do Patients Hospitalized With COPD Have Airflow Obstruction? Chest. 2017;151:1263-71.

16 Ulmer WT. Lung function-clinical importance, problems, and new results.[J]. J PHYSIOL PHARMACOL, 2003, 54 Suppl 1(2):11.

17 Vapnik V N. The nature of statistical learning theory [M]. New York: Springer, 2000: 138-167.

18 GLI-2012 desktop software for individual calculations. http://www.erseducation.org/guidelines/global-lung-function-initiative/spirometry-tools/desktop-individualcalculator.aspx. Last access Jun 3rd 2018.

19 Donner C F , Carone M , Bertolotti G , et al. Methods of assessment of quality of life[J]. Eur Respir Rev, 1997, 7(42):43-45.

20 Manhire A , Charig M , Clelland C , et al. Guidelines for radiologically guided lung biopsy[J]. Thorax, 2003, 58(11):920-36.

21 Berge M V D , Hop W C , Molen T V D , et al. Prediction and course of symptoms and lung function around an exacerbation in chronic obstructive pulmonary disease[J]. Respir Res, 2012, 13(1):44.

22 Celli BR, Thomas NE, Anderson JA, et al. Effect of Pharmacotherapy on Rate of Decline of Lung Function in Chronic Obstructive Pulmonary Disease [J]. Am J Respir Crit Care Med, 2008, 178(4):332-338. 
23 Torun E , Cakir E, Özgüç, Fatma, et al. The Effect of Obesity Degree on Childhood Pulmonary Function Tests[J]. Balkan Med J, 2014, 31(3):235-238.

24 Jing C,Yang Z, Qun Y et al. Prediction models for pulmonary function during acute exacerbation of chronic obstructive pulmonary disease [J]. Physiol Meas, 2020, 41, 125010.

25 Lonergan, M., Dicker, A.J., Crichton, M.L. et al. Blood neutrophil counts are associated with exacerbation frequency and mortality in COPD[J]. Respir Res, 2020, 21: 166.

26 Milara J, Juan G, Peiro T, Serrano A, Cortijo J. Neutrophil activation in severe, early-onset COPD patients versus healthy non-smoker subjects in vitro: effects of antioxidant therapy. Respiration. 2012;83(2):147-58.

27 Jones AW, Robinson R, Mohamed P, Davison G, Izzat HJ, Lewis KE. Impaired blood neutrophil function in the frequent Exacerbator of chronic obstructive pulmonary disease: a proof-of-concept study. Lung. 2016;194(6):881-7.

28 Corhay J , Moermans C, Henket M , et al. Increased of exhaled breath condensate neutrophil chemotaxis in acute exacerbation of COPD[J]. Respir Res, 2014, 15(1):115.

29 Perera WR, Hurst JR, Wilkinson TM, et al. Inflammatory changes, recovery and recurrence at COPD exacerbation. Eur Respir J. 2007;29(3):527-34.

30 Vapnik V N. The nature of statistical learning theory [M]. New York: Springer, 2000: 138-167.

31 Cristóbal, Esteban, Inmaculada, et al. A decision tree to assess short-term mortality after an emergency department visit for an exacerbation of COPD: a cohort study[J]. Respir Res, 2015.

32 Godtfredsen N S , Jrgensen D V , Marsaa K, et al. Soluble urokinase plasminogen activator receptor predicts mortality in exacerbated COPD[J]. Respir Res, 2018, 19(1):97.

\section{Figures}


Subjects enrolled from January 2018 to March 2020

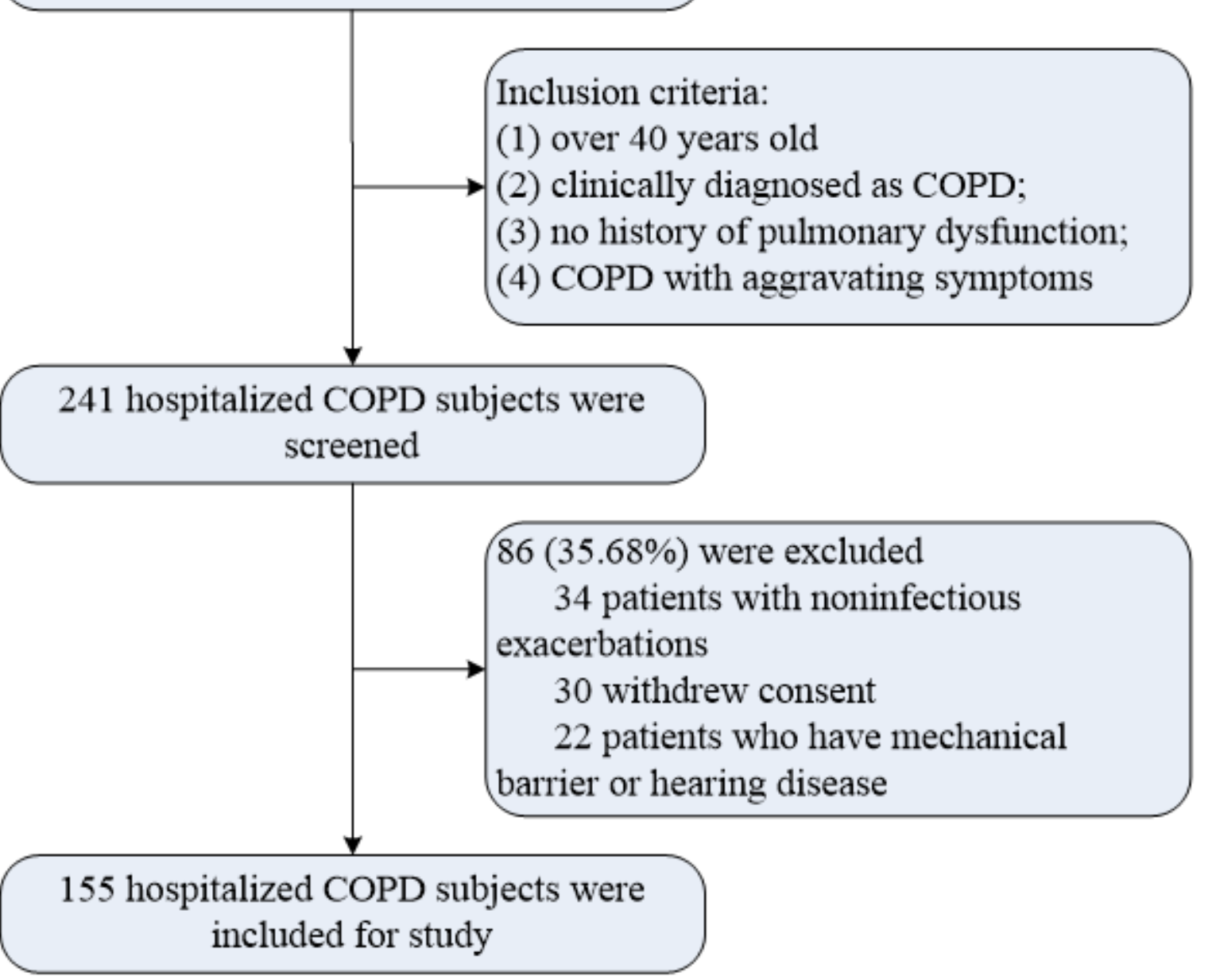

Figure 1

Flowchart of inclusion and exclusion criteria. 


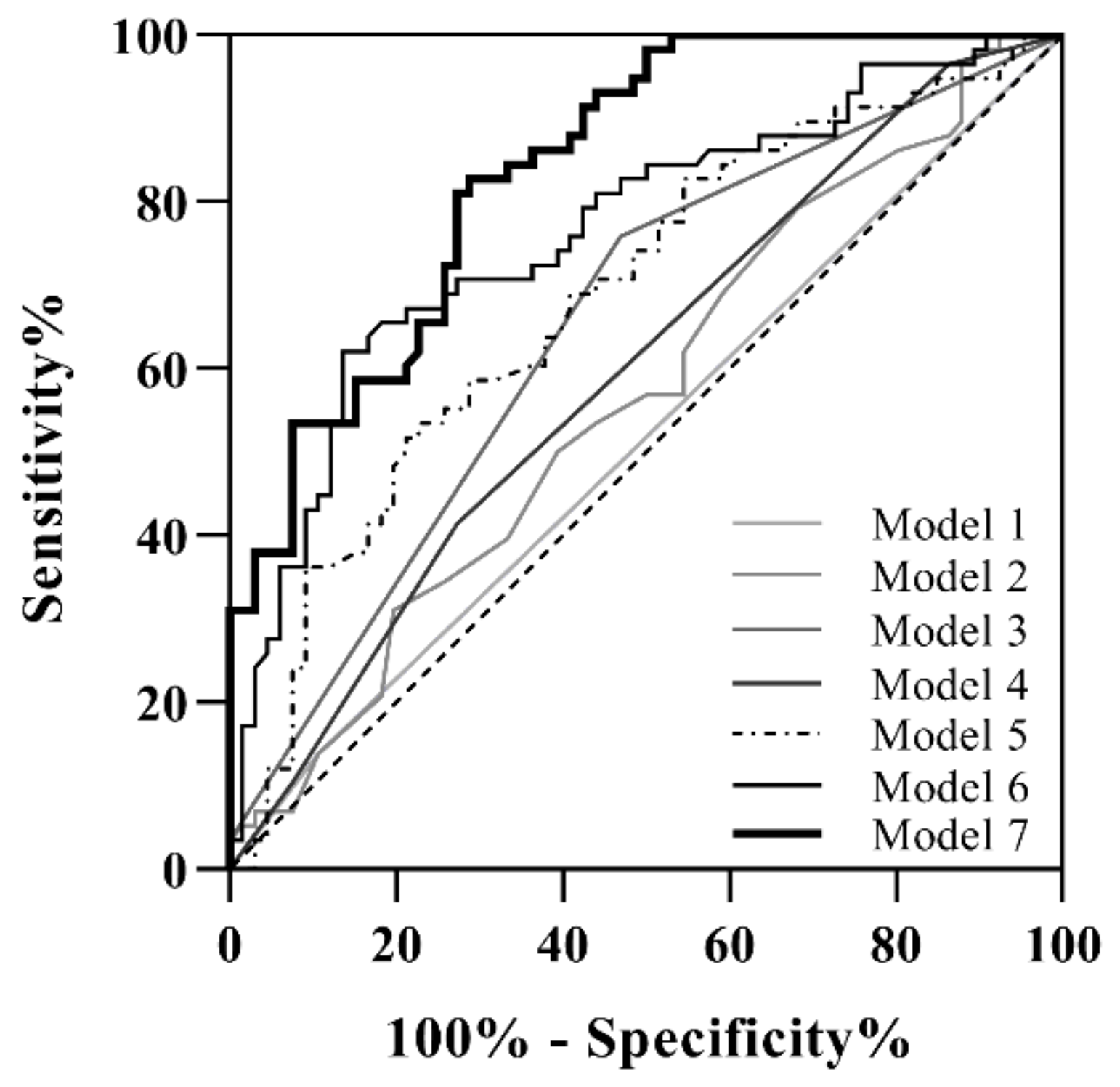

Figure 2

ROC curves demonstrating the ability of univariable and multivariable models to predict severe episode of ECOPD. Model 1=gender; Model 2=age; Model 3=weight; Model 4=BMl; Model 5=NEU \%; Model 6= multivariable model without NEU\%; Model 7= multivariable model. 


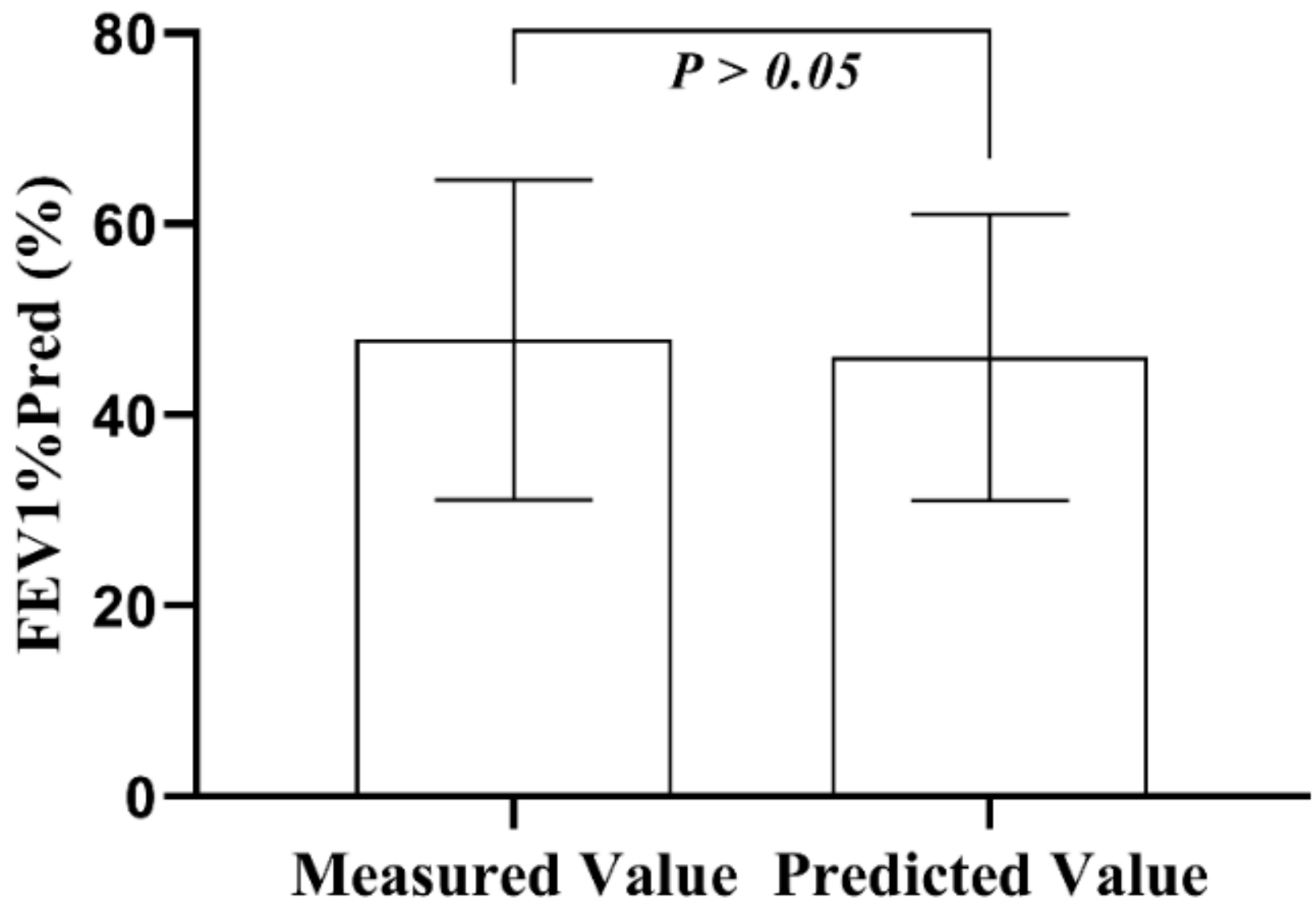

Figure 3

Comparison between the predicted and the measured FEV1\%pred value 


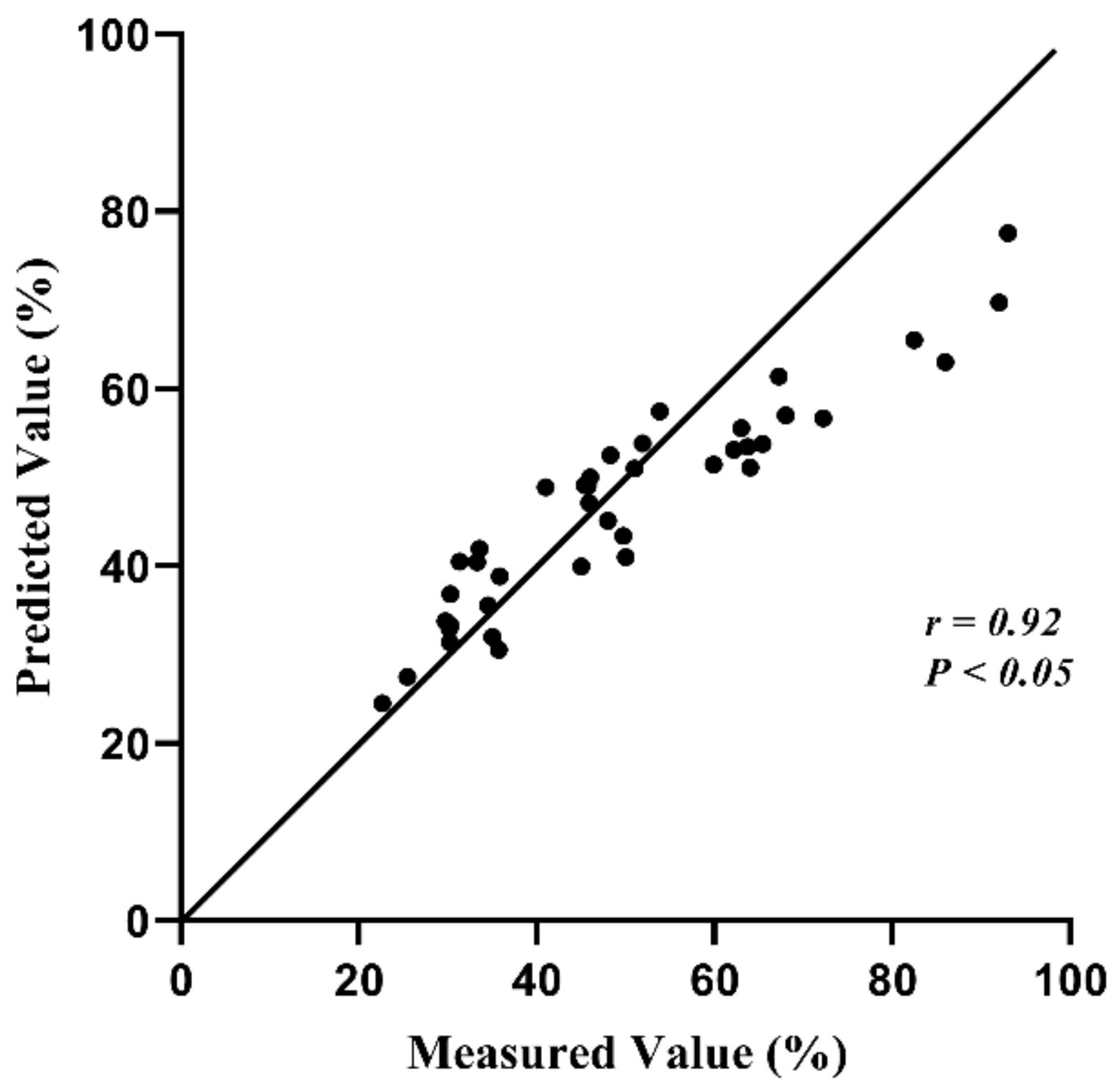

Figure 4

Linear relationship between the predicted and the measured FEV1\%pred value 


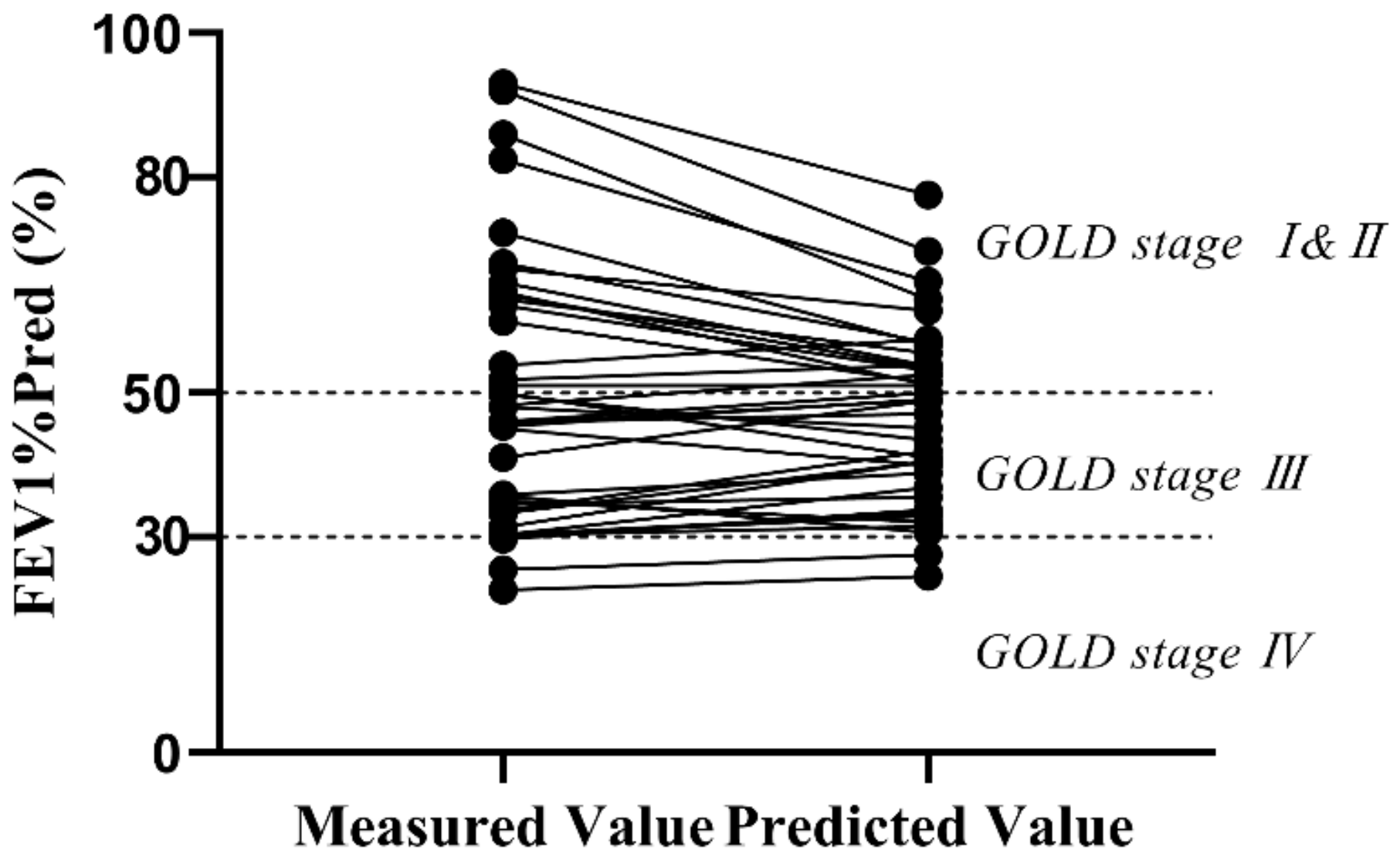

Figure 5

Comparison between the predicted and the measured GOLD stage, GOLD stage I and GOLD stage II were combined as the moderate group 